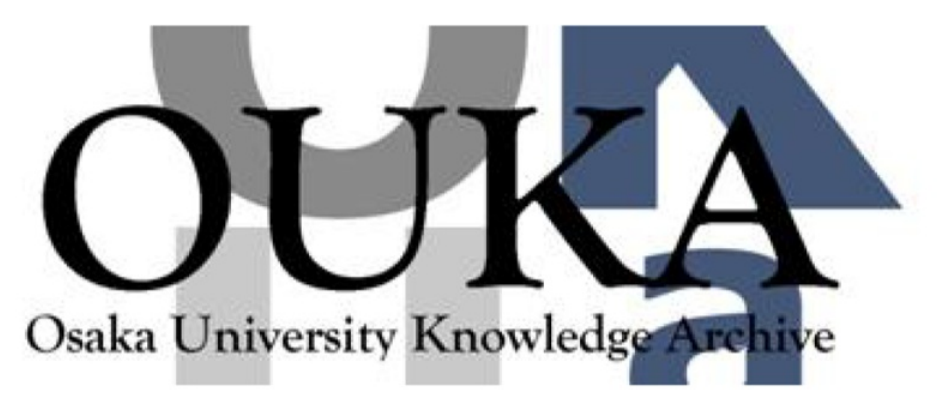

\begin{tabular}{|c|l|}
\hline Title & $\begin{array}{l}\text { Time-resolved study of polaron pairs in } \\
\text { conjugated polymers by two-correlated-pulses } \\
\text { technique }\end{array}$ \\
\hline Author(s) & $\begin{array}{l}\text { Frankevich, Eugene; Nishihara, Yusuke; Fuji i, } \\
\text { Akihiko et al. }\end{array}$ \\
\hline Citation & Physical Review B. 66(15) p. 155203-p. 155203 \\
\hline Issue Date & $2002-10-04$ \\
\hline oaire:version & VoR \\
\hline URL & https://hdl.handle. net/11094/75675 \\
\hline rights & $\begin{array}{l}\text { Copyright (2002) by the American Physical } \\
\text { Society }\end{array}$ \\
\hline Note & \\
\hline
\end{tabular}

Osaka University Knowledge Archive : OUKA

https://ir. Library. osaka-u. ac. jp/

Osaka University 


\title{
Time-resolved study of polaron pairs in conjugated polymers by two-correlated-pulses technique
}

\author{
Eugene Frankevich \\ Department of Electronic Engineering, Graduate School of Engineering, Osaka University, 2-1 Yamada-oka, Suita, Osaka 565-0871, \\ Japan \\ and Institute for Energy Problems of Chemical Physics, Russian Academy of Sciences, Leninskiy prospect 38-2, Moscow 117829, Russia \\ Yusuke Nishihara, Akihiko Fujii, Masanori Ozaki, and Katsumi Yoshino \\ Department of Electronic Engineering, Graduate School of Engineering, Osaka University, 2-1 Yamada-oka, Suita, Osaka 565-0871, \\ Japan
}

(Received 8 March 2002; revised manuscript received 21 June 2002; published 4 October 2002)

\begin{abstract}
Formation of polaron pairs is studied in photoconducting conjugated polymers such as substituted polythiophenes. The polaron pairs show themselves in the second order processes that were the main supplier of free charge carrier at high excitation density provided by femtosecond laser pulses. Two-correlated-pulses technique are used, and permitted to resolve kinetics of the free charge carrier formation within the picosecond time domain. The external electrical field is shown to change a relative amount of free charge carriers formed in the first and second order processes (i.e., by dissociation of primarily formed polaron pairs and by dissociation of the pairs formed as a result of recombination of primary pairs). A method of estimation of intercharge distances in the pairs based on the action of the electrical field is suggested and shows that polaron pairs of two intercharge distances (about 4 and $10 \mathrm{~nm}$ ) are formed at the photoexcitation of polythiophenes studied.
\end{abstract}

DOI: 10.1103/PhysRevB.66.155203

PACS number(s): 73.50.Pz, 72.80.Le, 71.35.-y, 78.47.+p

\section{INTRODUCTION}

Photoconductivity of conjugated polymers finds many applications. Understanding the mechanism of the photoconductivity is necessary for working under conditions of a high quantum yield of charge carriers. Many works were devoted to investigation of processes responsible for appearance of charged species under photoexcitation (see review papers in Ref. 1). Most of them came to the conclusion that charge carriers appear immediately as a result of the light absorption, though it remains uncertain whether the corresponding process can be described as band-to-band transition, or as a fast electron-transfer process from primarily generated intrachain excitons. Thus, for polymers studied in the present work that are substituted polythiophene the pump-and-probe technique have revealed that transient absorption clearly shows appearance of charged species during time interval shorter than 1 ps after the light absorption, ${ }^{2,3}$ though authors could not determine whether the charges belonged to polaron pairs or free polarons. Appearance of charge carriers under photoexcitation is a necessary but not sufficient condition for their taking part in the cw conductivity. For that they must be free to drift under the action of an electric field, and be shifted by the field during their lifetime. However, positive and negative charge carriers in molecular solids and polymers being formed in pairs remain in the vicinity of each other during all their lifetime and feel an attractive action of Coulomb field. ${ }^{4-7}$ That is a sequence of very fast energy loss by the charge carriers that is reflected in their low mobility values. They remain within a sphere limited by so-called Onsager's radius $r_{\mathrm{Ons}}=e^{2} / \epsilon k T$ (typical value at room temperature being about $15 \mathrm{~nm}$ ) and recombine geminately, leaving zero net contribution to the $\mathrm{cw}$ conductivity. A small part of the pairs manages to dissociate and contribute to the conductivity. The pairs can show themselves in the transient conductivity measurements as real charge carriers, but they subtract the contribution to the photocurrent later on when recombining geminately. ${ }^{8}$ In order to be sure that one deals with really free charge carrier one has to measure photocurrent during the time interval longer than lifetime of the pairs. Such approach was realized in works ${ }^{9,12}$ where average laserpulse-induced photoconductivity measured, and time resolution was achieved due to changing of parameters of the laser pulses.

Dissociation of the pairs does not appear to be the only way of formation of free charge carriers. They can be formed also as a result of interpair recombination: two charges from neighbor pairs recombine just leaving two remaining charges at higher intercharge distance and thus increasing their chance to be outside Onsager's radius and get free. This idea was considered in Ref. 11 in application to quasi-onedimensional semiconductors such as polydiacetylene, and permitted to rationalize a superlinear dependence of the laser pulse induced photocurrent in PPV on the light intensity in the work. ${ }^{9}$

In the present work, we studied processes responsible for the formation of free polarons in conjugated polymers under laser light (400 nm wavelength) excitation by the new technique based on the correlated action of two 150 fs laser pulses with controlled delay time between them. There is a feature in the action, which changes the yield of the products of the second order processes as a function of the delay time under conditions of the constant average intensity of the laser excitation. The technique permitted to get direct evidence on recombination processes responsible for free polaron formation and to measure the lifetime of the species involved. A scenario of the processes and equations describing them involve primary formation of pretrapped polaron pairs, termalization of the pairs, dissociation, and interpair recombination. 
We have paid attention in the present work to the competition in the free carrier formation between first- and secondorder processes. That competition is shown to be varied by changing the strength of the electrical field, which affected the dissociation rate of polaron pairs (the first order process). The sensitivity of the processes involved to the external electrical field serves as additional evidence of involvement of polaron pairs into the play. A signal measured by a twocorrelated-pulse technique versus the electrical field strength permitted us then to find out the changes of the free carrier yield connected solely with the dissociation of the pairs, and estimate initial interpolaron distances in the pairs.

\section{METHODICAL}

For studying second order processes responsible for the formation of free charge carriers we applied a twocorrelated-pulse laser technique. ${ }^{10,12} \mathrm{~A}$ pretext for it was the observation of superlinear dependence of the prompt component of the photoconductivity on the intensity of the laser pulses. ${ }^{9}$ We suggested to split the pulses generated by the laser into pairs of pulses with controlled delay time between them and illuminate the sample by these correlated pulses. ${ }^{10}$ Though the total intensity of the light remained the same at any delay time, the yield of any products of second order processes would depend on the density of species taking part in the processes. The density itself depends on superposition of the species produced by the first pulses of the pairs and survived up to the moment of action of the second pulses. The rate of formation $U$ of products of a second order process is expected to depend on the delay time $\Delta t$ and the intensity of equal light pulses $I$ as

$$
U \propto I^{2}[1+f(\Delta t)] .
$$

Here $f(\Delta t)$ is the function normalized to unity and describing the decay of species taking part in the second order process. This formula appears as the result of integration of the products of a bimolecular reaction between intermediate species generated by two short pulses with intensities $I_{1}$ and $I_{2}$, the second one being delayed by $\Delta t$ in respect to the first one. In the case of $f(t)$ having exponential view with $k_{1}$ as a monomolecular decay rate constant the integration looks similar to

$$
\begin{aligned}
U & \propto \int_{0}^{\Delta t}\left(I_{1} e^{-k_{1} t}\right)^{2} d t+\int_{\Delta t}^{\infty}\left(I_{2} e^{-k_{1}(t-\Delta t)}+I_{1} e^{-k_{1} t}\right)^{2} d t \\
& =I_{1}^{2}+I_{2}^{2}+2 I_{1} I_{2} e^{-k_{1} \Delta t} .
\end{aligned}
$$

The technique can be applied as to second order processes responsible for formation of products, and to recombination processes that lead to disappearance of final products. Next scheme may serve as an example of possibilities of the technique.

Let us consider as an example formation and recombination of species $M^{*}$ in the next sequence of processes. Primary excitation

$$
h \nu+M \Rightarrow M^{*} .
$$

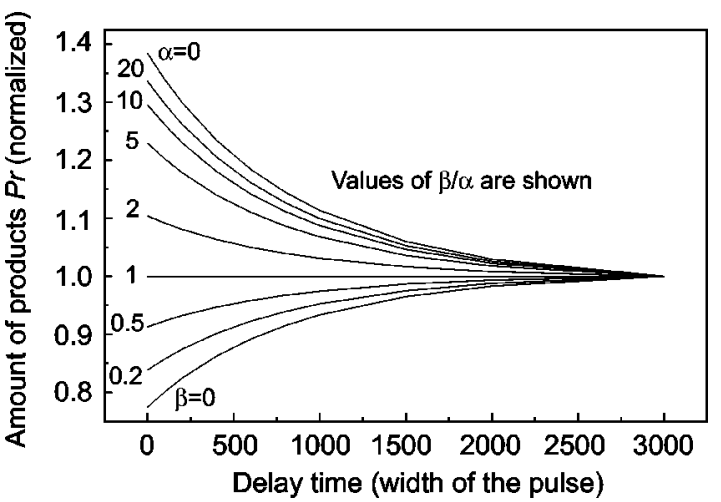

FIG. 1. Total yield of products of model processes (3)-(5) as a function of delay time between pulses. The curves were calculated by computer for the system of differential equations (6) for different ratios of parameters $\alpha$ and $\beta$.

Bimolecular annihilation process (rate constant $k_{2}$ ) and formation of a product $\operatorname{Pr}_{2}$ with a probability $\beta$

$$
M^{*}+M^{*} \Rightarrow \operatorname{Pr}_{2}
$$

Monomolecular decay (rate constant $k_{1}$ ) and formation of a product $\operatorname{Pr}_{1}$ with a probability $\alpha$

$$
M^{*} \Rightarrow M ; \quad M^{*} \Rightarrow \operatorname{Pr}_{1}
$$

One can monitor just the sum of the products $\operatorname{Pr}$ if $\operatorname{Pr}_{1}$ and $\mathrm{Pr}_{2}$ are of the same nature. It is just the case when the products are charge carriers.

Kinetics of the above processes can be described by the next equations, the first of them dealing with a process of formation of species $M^{*}$ initiated by two-pulse excitation and their decay; the last equation corresponds to accumulation of the products $\mathrm{Pr}_{1}$ and $\mathrm{Pr}_{2}$, formed in the processes (4) and (5) with probabilities $\alpha$ and $\beta$ :

$$
\begin{gathered}
\frac{d M^{*}}{d t}=g(t, \Delta t)-k_{1} M^{*}-k_{2} M^{* 2}, \\
\frac{d \operatorname{Pr}}{d t}=\alpha k_{1} M^{*}+\beta k_{2} M^{* 2} .
\end{gathered}
$$

Here $g(t, \Delta t)=g_{1} \exp \left[-(t-\Delta t)^{2} / t_{0}^{2}\right]+g_{2} \exp \left(-t^{2} / t_{0}^{2}\right)$ is the rate of formation of primary species $M^{*}$ by two pulses having duration $t_{0} ; g_{1}$ and $g_{2}$ are maximum rates of formation by the delayed and undelayed pulses; $\Delta t$ is a delay time between the pulses; $k_{1}$ and $k_{2}$ are first and second order rate constants. Figure 1 shows dependencies of the total amount of products Pr, which can be monitored in an experiment, on the delay time $\Delta t$. The curves were calculated for different ratios of parameters $\alpha$ and $\beta$. Falling curves indicate the bimolecular process of the formation of products $\mathrm{Pr}_{2}$ to prevail, while the rising curves do for the monomolecular process to be responsible mainly for the products. When the ratio of the product formation rates becomes equal to that of terms $k_{1} M^{*}$ and $k_{2} M^{* 2}$ (it means that $\alpha=\beta$ ), the yield of products does not depend on the delay time. The curves were calculated for the conditional values of parameters $t_{0}=1$, 
$g_{0}=1, k_{1}=10^{-3}, k_{2}=10^{-3}$. Integration was carried out up to times $t=10^{4} t_{0}$, which were much longer than lifetime of $M^{*}\left(1 / k_{1}\right)$.

In the present work we changed the values of parameters $\alpha$ and $\beta$ artificially by external electrical field. As far as we assume that we are dealing with recombination of polaron pairs, then one can consider $M^{*}$ as a polaron pair, and parameters $\alpha$ and $\beta$ as those connected with probabilities of dissociation of primarily formed polaron pairs, and the pairs formed as a result of recombination.

\section{EXPERIMENTAL}

In the present work, we studied alkyl-substituted polythiophenes, poly(3-octylthiophene) (PAT8), and poly(3dodecylthiophene) (PAT12). The polymers were synthesized and purified by the methods already reported. ${ }^{13}$

Films of polymers were prepared by casting from toluene solution on the quartz substrates with comblike patterned interdigital $\mathrm{Au} / \mathrm{Cr}$ electrodes of $10 \mu \mathrm{m}$ width separated by a distance of $5 \mu \mathrm{m}$. Sandwich type samples were prepared as well between ITO and $\mathrm{Al}$ electrodes. The thickness of polymer film was about a few $\mu \mathrm{m}$. The experimental setup was the same as used in the work. ${ }^{12}$ Electrical part was able to measure the modulated average photocurrent $\left(f_{\bmod }=20\right.$ to $100 \mathrm{~Hz}$ ) by the lock-in amplifier (Stanford Research System SR830) $(\mathrm{RC}=0.01 \mathrm{~s})$. The sample was put in the vacuum chamber $\left(10^{-5}\right.$ Torr $)$ at room temperature.

For excitation source, a Ti:sapphire laser based regenerative amplifier system was used. The pulse width, wavelength and pulse repetition rate of the output laser beam were 150 fs, $800 \mathrm{~nm}$, and $1 \mathrm{kHz}$, respectively. The beam were frequency doubled by a $1-\mathrm{mm}$ thick BBO crystal to obtain a laser beam with the wavelength of $400 \mathrm{~nm}$. The beam was then divided into two parts by a beam splitter. Both parts were focused into the sample. One beam was delayed by using a computer controlled delay line. Delay time can be variably changed between 0.03 ps to $1 \mathrm{~ns}$. Pulse energies were measured, and the light intensity at the sample position can be varied within the range from 1 to $10 \mu \mathrm{J}$ per pulse. The illumination area at the sample was about $0.04 \mathrm{~cm}^{2}$.

In order to increase the sensitivity of the technique, we modulated the sequence of the first (undelayed) pulses by a chopper with a low frequency. A lock-in amplifier measured the amplitude of the modulated photocurrent and its phase shift at low modulation frequency while changing the delay time. The modulated signal was proportional to the difference between signals produced by two sequences of pulses: undelayed and delayed pulses acting together and delayed pulses acting alone.

\section{RESULTS}

We measured an average photocurrent vs. delay time at different bias voltages covering the electrical field strength from $10^{3}$ to $4 \times 10^{5} \mathrm{~V} / \mathrm{cm}$. A typical curve obtained on a PAT8 regiorandom sample in the first-pulse-chopped regime is presented in Fig. 2. A sandwich-type sample was used in this experiment.

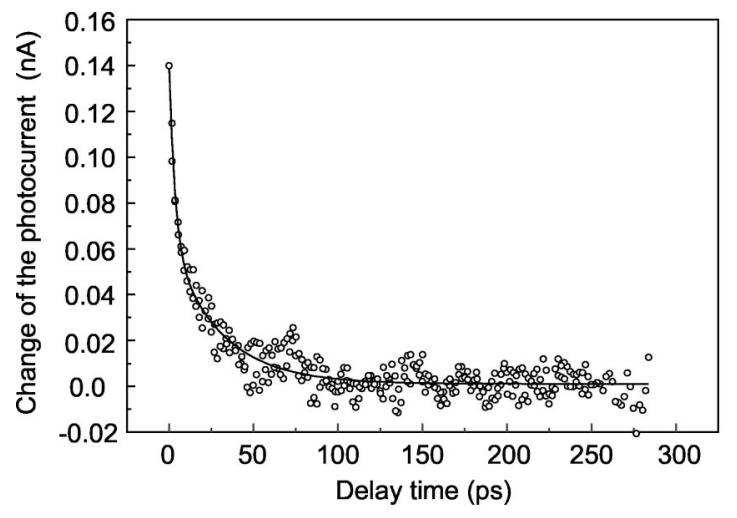

FIG. 2. Dependence of the average modulated photocurrent measured in the two-correlated-pulse regime on the delay time between the pulses. Sample PAT8 of the sandwich type between ITO and $\mathrm{Al}$ electrodes; light intensity is $2.1 \mu \mathrm{J} /$ pulse per $4 \mathrm{~mm}^{2}$. Solid curve is a fitting of experimental points by computer calculation of the system of equations (7).

We estimated the value of the average photocurrent that could be expected at the excitation of our sample by the laser light depositing an energy of about $25 \mu \mathrm{J} / \mathrm{cm}^{2}$ per a $150 \mathrm{fs}$ pulse ( $1 \mu \mathrm{J}$ pulses $), 10^{3}$ times per second. Assuming a free charge carrier quantum yield $\beta=10^{-2}$, extinction coefficient $\kappa=5 \times 10^{4} \mathrm{~cm}^{-1}$, mobility of free charge carriers $\mu$ $=10^{-5} \mathrm{~cm}^{2} / \mathrm{Vs}$, which correponds to the rate constant for free charge carrier recombination $k_{\text {rec }}=6 \times 10^{-12} \mathrm{~cm}^{3} / \mathrm{s}$, taking into account recombination of free charge carriers, and using geometry parameters of the samples we arrived at the following estimations.

Average density of charge carriers $n_{a v}=8 \times 10^{14} 1 / \mathrm{cm}^{3}$; photocurrent averaged for the pulse repetition period $i_{\mathrm{ph}}$ $\approx 10^{-10} \mathrm{~A}$ at the field strength $E=2 \times 10^{4} \mathrm{~V} / \mathrm{cm}$. This coincides with the photocurrent observed experimantally within an order of magnitude.

We have shown in the methodical part that the relative magnitude of the delay-time-dependent photocurrent is very sensitive to relative values of coefficients before first- and second-order terms $(\alpha$ and $\beta$ ). As far as we assume those terms are related to polaron pairs the coefficients must be sensitive to electrical field strength. In order to check that, and to study an effect of electrical field strength $E$ on dissociation rate of polaron pairs we measured dependences on $E$ of the value $R$, which is the ratio of the average photocurrents registered at zero delay time, and at the delay time much higher than the width of the decaying curve shown in Fig. 2: $R=i_{\mathrm{ph}}(\Delta t=0) / i_{\mathrm{ph}}(\Delta t=500 \mathrm{ps})$.

The ratio $R$ depends on the laser light power and tends to $R=1$ at low intensities. Changing the light intensity led to a related change of the $R$ value, but did not affected the lifetimes of polaron pairs, thus showing that the pairs dissapeared mainly in the first order processes.

Figures 3-5 show the results obtained. One can see that electrical field really affects the $R$ value, and typically $R(E)$ plots have two maximums.

\section{DISCUSSION}

For description of the processes responsible for the photoconductivity we have used a system (7) of kinetic equa- 


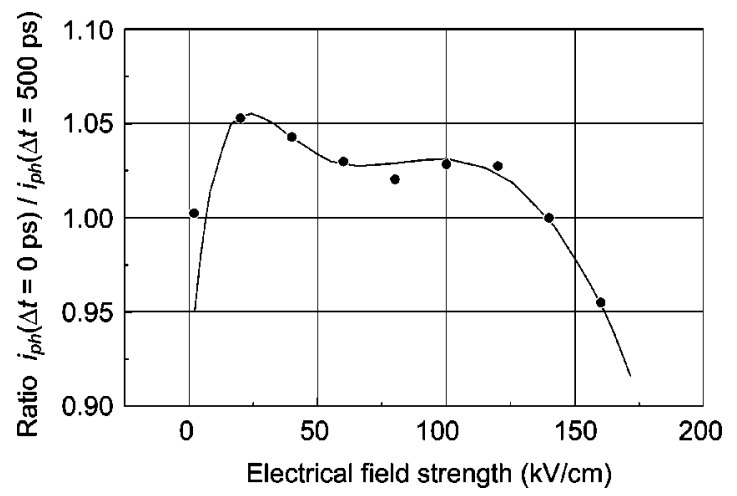

FIG. 3. Dependence of the ratio $R=i_{\mathrm{ph}}(\Delta t=0) / i_{\mathrm{ph}}(\Delta t$ $=500 \mathrm{ps})$ on the electrical field strength $E$. PAT8 sample, interdigital electrodes, light power is $8 \mu \mathrm{J} /$ pulse. Solid line is fitting by Eq. (12).

tions, corresponding to formation of pretrapped $\left(P_{1}\right)$ and trapped $\left(P_{2}\right)$ polaron pairs, and free charge carriers $(p)$. The existence of pretrapped pairs was substantiated earlier: ${ }^{9}$

$$
\begin{aligned}
& \frac{d P_{1}}{d t}=g(t, \Delta t)-k_{11} P_{1}-k_{21} P_{1}^{2}-k_{212} P_{1} P_{2}, \\
& \frac{d P_{2}}{d t}=k_{l o c} P_{1}-k_{12} P_{2}-k_{22} P_{2}^{2}-k_{212} P_{1} P_{2}, \\
& \frac{d p}{d t}=\alpha_{1} k_{11} P_{1}+\alpha_{2} k_{12} P_{2}+\beta_{1} k_{21} P_{1}^{2}+\beta_{2} k_{22} P_{2}^{2} \\
& \quad+\beta_{3} k_{212} P_{1} P_{2} .
\end{aligned}
$$

Here $g(t, \Delta t)$ is the rate of formation of polaron pairs $P_{1}$. In the two-pulses-correlation regime $g(t, \Delta t)=g_{0}[\exp \{-(t$ $\left.\left.-\Delta t)^{2} / t_{0}^{2}\right\}+\exp \left\{-\left(t / t_{0}\right)^{2}\right\}\right]$, where $t_{0}$ is the light pulse width, $P_{1}$ and $P_{2}$ are densities of primary (pretrapped) and thermalized polaron pairs, respectively, $k_{11}$ and $k_{12}$ are their monomolecular rate constants (for geminate recombination, dissociation, and localization), $k_{21}, k_{212}$, and $k_{22}$ are rate constants for random interpair recombination, and $k_{\text {loc }}$ is the thermalization rate constant for pretrapped polaron pairs. The

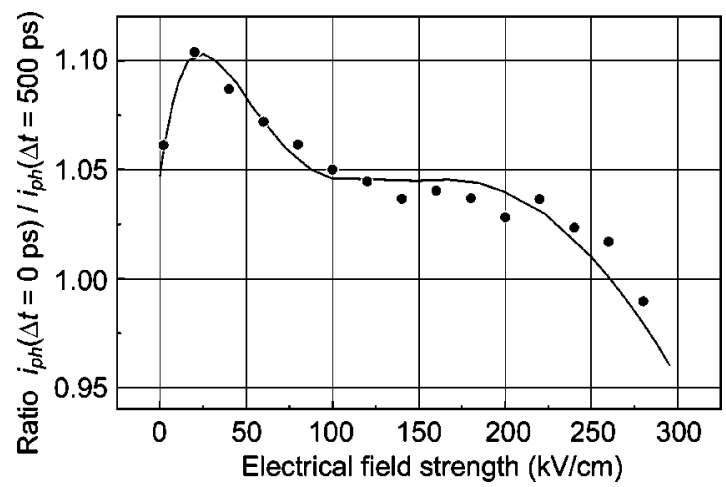

FIG. 4. Dependence of the ratio $R=i_{\mathrm{ph}}(\Delta t=0) / i_{\mathrm{ph}}(\Delta t$ $=500 \mathrm{ps})$ on the electrical field strength $E$. PAT12 sample, interdigital electrodes, light power is $8 \mu \mathrm{J} /$ pulse. Solid line is fitting by Eq. (12).

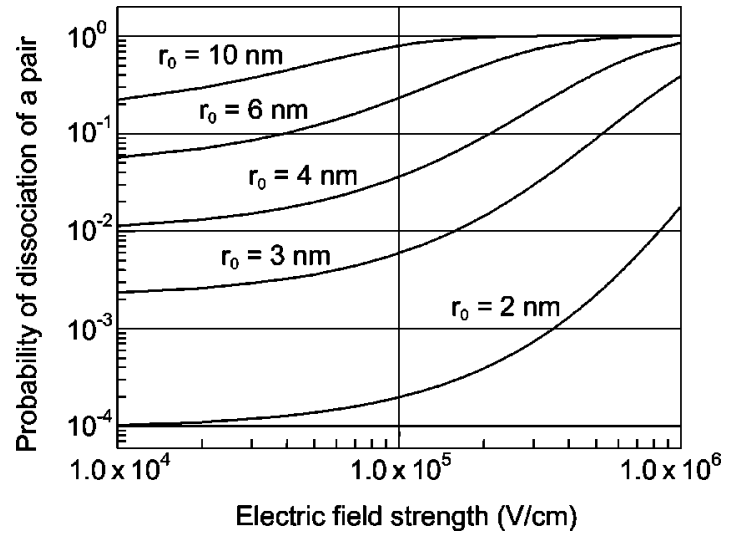

FIG. 5. Probability of dissociation as a function of electrical field strength for polaron pairs with different intercharge distances. Curves are calculated by the formula (8) at $\epsilon=3$ and $T=300 \mathrm{~K}$.

last equation of the system (7) describes accumulation of free polarons originated from five sources, namely, (i) and (ii) field-assisted dissociation of thermalized polaron pairs, (iii) to $(\mathrm{v})$ recombination of pretrapped pairs, thermalized pairs, and recombination between two different kinds of pairs. Values $\alpha_{i}$ and $\beta_{i}$ are probabilities of free polaron formation in those processes. We integrated the system of equations (7) by computer over the time interval much longer than the lifetime of any species taking part in the processes (up to $1.5 \mathrm{~ns}$ ) and have got the dependencies of amount of free charge carriers accumulated $p$ on the delay time $\Delta t$. The average photocurrent measured is believed to be proportional to that amount, though possible bulk recombination of free charge carrier can distort this proportionality a little bit. Though a big number of parameters in the system do not permit to insist on the values chosen to be unique, there is no big choice of the main parameters within the general scheme chosen that are able to describe general features of the experimental curves. While fitting the experimental curve shown in Fig. 2 we neglected by recombination between $P_{1}$ and $P_{2}$ and have used the following values: $g_{0}=3.3$ $\times 10^{30} \mathrm{~cm}^{-3} \mathrm{~s}^{-1}, \quad k_{11}=2.9 \times 10^{11} \mathrm{~s}^{-1}, k_{21}=10^{-7} \mathrm{~cm}^{3} \mathrm{~s}^{-1}$, $k_{\text {loc }}=1.3 \times 10^{11} \mathrm{~s}^{-1}, \quad k_{12}=3.3 \times 10^{10} \mathrm{~s}^{-1}, \quad k_{22}=6.7$ $\times 10^{-8} \mathrm{~cm}^{3} \mathrm{~s}^{-1}, \beta_{1}=\beta_{2}=10^{-1}$, and $\alpha_{1}=\alpha_{2}=10^{-2}$. The fitting shows that the lifetimes of the species involved in the processes are about 3 ps (for pretrapped polaron pairs) and 30 ps (for thermalized polaron pairs). The same lifetimes can be obtained by fitting $i_{\mathrm{ph}}(\Delta t)$ curve by a sum of two exponents.

The ratio $R$ depends on the laser light power and tends to $R=1$ at low intensities. We were working at the laser pulse intensity when values of the first- and second-order terms in the last Eqs. (7) were comparable with each other that was reflected by crossing the line $R=1$ at low and high electrical fields (Figs. 3 and 4).

The technique supplies a unique possibility to check our assumption about involvement of polaron pairs into the first and second order processes studied. It is based on the dependence of the magnitude of the ratio $R=p(\Delta t=0) / p(\Delta t$ $\gg 0 \mathrm{ps}$ ) on the relative value of the coefficients $\alpha$ and $\beta$, as was shown in the methodical part of this paper. It is possible 
for polaron pairs only to vary these coefficients just changing the electrical field strength $E$ not going to very high field strengths.

It is known as well that electrical field can induce dissociation of primarily formed singlet excitons, as it is shown in the pape ${ }^{14}$ by studying the electrical field induced quenching of the fluorescence. Polaron pairs, however, require for their dissociation a field strength much lower than that needed for singlet exciton dissociation. Effective dissociation of singlet excitons starts at the electrical field strength higher than $10^{6} \mathrm{~V} / \mathrm{cm} \quad(10 \%$ fluorescence quenching at that field strength $^{14}$ ), while an order of magnitude lower strength causes about complete dissociation of polaron pairs.

Let us consider what one can expect within the framework of two different models.

(1) Free polarons $p$ are produced from two sources, namely, by dissociation of thermalized polaron pairs, which have an intercharge distance $r_{01}$, and directly by recombination of these pairs. This means that $\alpha=\phi\left(r_{01}, E\right)$ and $\beta$ $=$ const.

(2) The value of the $R$ depends on the ratio of dissociation rate of a primarily formed polaron pairs $\left[\alpha=\phi\left(r_{01}, E\right)\right]$ and dissociation rate of new polaron pairs that appear as a result of interpair recombination of primary pairs. Intercharge distance $r_{02}$ in these new pairs may happen to be not big enough for immediate dissociation. One can expect the distance $r_{02}$ being about twice as big as initial separation of charges in primarily formed pairs is still shorter or comparable with Onsager's radius. In such a case the dissociation of new pairs will be electrical field sensitive as well, though obeying to a different low: $\beta \propto \phi\left(r_{02}, E\right)$.

One needs to choose a proper function describing the probability of dissociation of polaron pairs. That was done first by Onsager, ${ }^{15}$ and applied later on to dissociation of polaron pairs in organic semiconductors ${ }^{16,17}$ and polymers. ${ }^{18}$ For the purpose of estimations we are using here a model based on the dissociation of pairs by overcoming a potential barrier $\Delta U=e^{2} / \epsilon r_{0}$, where $r_{0}$ is an average inter-charge distance in a pair. This barrier becomes lower at higher electrical field and disappears at Eer $>\Delta U$. We suggest describing the probability of dissociation as a function of the electrical field strength $E$ and intercharge distance $r_{0}$ by the formula

$$
\phi\left(r_{0}, E\right) \propto \exp \left\{-\frac{e^{2}}{\epsilon r_{0} k T} \exp \left(-\frac{E \epsilon r_{0}^{2}}{e}\right)\right\} .
$$

This formula gives the dependence $\phi\left(r_{0}, E\right)$, which is shown in the Fig. 6, and it coincides exactly with that calculated in Ref. 18 by the Onsager's formula for dissociation of geminate pairs, ${ }^{15}$ but is much more convenient for analytical treatment. A similar formula is believed to be valid for dissociation of the pairs, which appear as a result of interpair recombination. The difference is because an increased value of interpolaron distance $r_{02}=S r_{01}(S>1)$ is inserted.

The results of computer calculation of the $R$ ratio according to models (1) and (2), based on the system of equations (7), are presented in Fig. 6. The curves were computer calculated from the kinetic equations (7) at the next values of

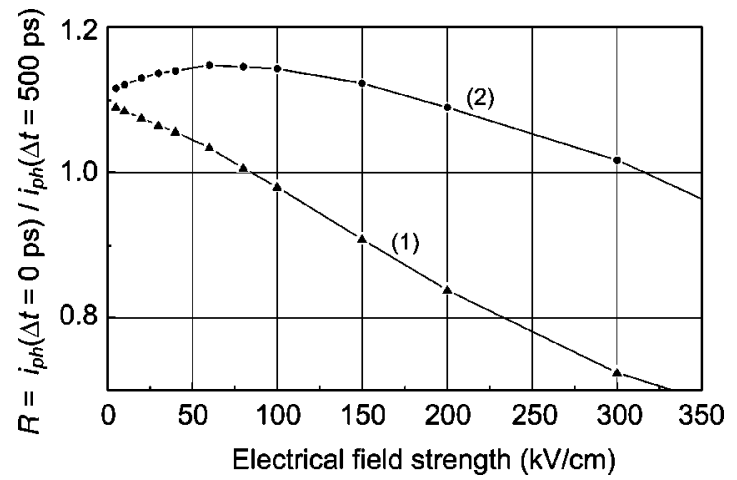

FIG. 6. Dependencies $R(E)$ for two models of free polarons formation. The curve (1) is calculated at the assumption that free charge carriers are formed at the interpairs recombination independently of the electrical field strength. The curve (2) takes into account formation of new polaron pairs at the inter-recombination of primarily formed polaron pairs. See text for details.

the parameters $g=3.3 \times 10^{30} 1 / \mathrm{cm}^{3} \mathrm{~s}, \quad k_{11}=2.9 \times 10^{11} \mathrm{~s}^{-1}$, $k_{\text {loc }}=1.3 \times 10^{11} \mathrm{~s}^{-1}, \quad k_{12}=3.3 \times 10^{10} \mathrm{~s}^{-1}, \quad k_{21}$ $=10^{-7} \mathrm{~cm}^{3} \mathrm{~s}^{-1}$, and $k_{22}=6.7 \times 10^{-8} \mathrm{~cm}^{3} \mathrm{~s}^{-1}$.

We changed the coefficients $\alpha$ and $\beta$ in the last equation of the system (7) as a function of the field strength according to Eq. (8). Intercharge distances were chosen as $r_{01}=4 \mathrm{~nm}$ and $r_{02}=2 r_{01}$. The results of calculation show that one can expect monotonous decay of the $R$ ratio in case (1) and an appearance of a maximum in case (2). Position of the maximum is directly connected with intercharge distance $r_{01}$ and can be estimated by the formula (9), where parameter $S$ is the ratio $S=r_{02} / r_{01}$. Thus, at $r_{01}=4 \mathrm{~nm}$ and $S=2 E_{\max }=7$ $\times 10^{4} \mathrm{~V} / \mathrm{cm}$.

$$
E_{\max }(\mathrm{V} / \mathrm{cm})=\frac{e}{\epsilon r_{01}^{2}} \frac{\ln S}{\left(S^{2}-1\right)} \times 300
$$

One can see in Figs. 3-5 that experimentally the value of $R=i_{\mathrm{ph}}(\Delta t=0 \mathrm{ps}) / i_{\mathrm{ph}}(\Delta t=500 \mathrm{ps})$ depends on $E$ nonmonotonously, thus indicating that dissociation of both types of polaron pairs, primarily formed and born as a result of interpair recombination, depends on the electrical field strength, as in the model (2). However, a feature of the curves consists in having two maximums. We believe that this fact is a sequence of formation of two types of polaron pairs with different intercharge distances.

We tried modeling the situation, which might result in appearance of two maximums in the $R(E)$ curve. For that we have used a system of equations such as those shown above (7) for trapped polaron pairs, but with addition of new pairs and terms describing second order recombination, between pairs having different interpair distances.

The system of equations (7) considered second order processes as a reaction between movable species interacting with a rate constant $k_{2}$. However, one can imagine that even immovable species having a finite volume $\nu$ can take part in the second order processes during their accumulation just due to superposition of the volumes. Effective rate constant in such a case has a value $k_{2}=\nu / t_{0}$ and works only during 
the action of the light pulse. We suspect that the situation in respect to polaron pairs in conjugated polymers looks similar to that where a polaron pair can diffuse very slowly as an individual species. If so, one can construct an equation describing an amount of free charge carrier produced by the pairs of two sizes from five sources.

Dissociation of two types of primarily formed pairs

$$
\begin{aligned}
p_{1}(\Delta t=500 \mathrm{ps})= & G a\{1-2 G \nu a-2 G \nu(1-a)\} \\
& \times \phi\left(r_{1}, E\right)+G(1-a) \\
& \times\{1-2 G \nu(1-a)-2 G \nu a\} \phi\left(r_{2}, E\right) .
\end{aligned}
$$

Here $G$ is the density of primary pairs formed per a pulse, $a$ is a part of the primary pairs with an intercharge distance $r_{1}$, and the terms in brackets take into account reduction of the amount of primary pairs due to their superposition.

Dissociation of three types of secondary pairs formed as a result of interpair recombination, which occurs during the action of the light pulse

$$
\begin{aligned}
p_{2}(\Delta t=500 \mathrm{ps})= & G^{2} \nu a^{2} s \phi\left(2 r_{1}, E\right) \\
& +G^{2} \nu s(1-a)^{2} \phi\left(2 r_{2}, E\right) \\
& +G^{2} \nu a s(1-a) \phi\left(r_{1}+r_{2}, E\right) .
\end{aligned}
$$

Here $s$ is a parameter showing a maximum probability of formation of free polarons in result of interpair recombination. We neglected here by diminishing of amount of primary polaron pairs caused by their dissociation that may be important at highest electrical field strength. The sum of corresponding amounts of free polarons $p_{1}+p_{2}$ formed from these sources is considered as the value proportional to the photocurrent measured $i_{\mathrm{ph}}$.

An amount of free polarons formed in second order processes at $\Delta t=0$ is known to be twice as big as that at $\Delta t$ $=500 \mathrm{ps}$ [see Eq. (1)], amounts from first order processes remaining the same: $p_{2}(\Delta t=0)=2 p_{2}(\Delta t=500 \mathrm{ps}), p_{1}(\Delta t$ $=0)=p_{1}(\Delta t=500 \mathrm{ps})$. Thus the value of the ratio $R(E)$ can be expressed as

$$
R(E)=\frac{p_{1}(\Delta t=0)+p_{2}(\Delta t=0)}{p_{1}(\Delta t=500 \mathrm{ps})+p_{2}(\Delta t=500 \mathrm{ps})} .
$$

We made fitting of experimental curves $R(E)$ by Eq. (12) by choosing values of parameters $r_{1}, r_{2}, a, s$ and the yield of primary polaron pairs. Positions of maximum depend on values of $r_{1}$ and $r_{2}$; the amplitudes are sensitive to the value of $a$ and $s$. The yield of primarily formed pairs was taken as 0.2. One can see the results of fitting in Figs. 3 and 4 (solid curves) and below: PAT $8 r_{1}=4.1 \mathrm{~nm}, r_{2}=11 \mathrm{~nm} ; a$ $=0.937,1-a=0.063, s=0.33$, PAT $12 r_{1}=3.4 \mathrm{~nm}, r_{2}$ $=9 \mathrm{~nm} ; a=0.886,1-a=0.114, s=0.4$.

The structure of polythiopenes studied in the present work was investigated previously for crystalline samples. ${ }^{19}$ The results have shown that the interchain distance in the plane of thiophene rings increases with the length of alkyl chain, but separation between chains $(0.75 \mathrm{~nm})$, which have face-to-face arrangement does not strongly depend on the chain length up to $12 \mathrm{CH}$ groups. One can believe that effective conjugation length may be slightly larger than 10 thiophene rings (about $7 \mathrm{~nm}$ ). Results of the present paper show that the difference of intercharge distances for two types of thiophenes is relatively small, though the side chain lengths are much more different. It corresponds probably to the charge-transfer occurring mainly between face-to-face chains.

Thus, our estimation shows that primarily formed polaron pairs have inter-polaron distances about 4 and $10 \mathrm{~nm}$, and the pairs formed as a result of interpair recombination of polarons have an interpolaron distance about 8 and $20 \mathrm{~nm}$. These values have to be compared with Onsager's radius $r_{\text {Ons }}=e^{2} / \epsilon k T=18.5 \mathrm{~nm}$ at $\epsilon=3$ and room temperature. The comparison shows that polarons surviving interpair recombination remain inside of Coulomb well, though their chances to get free increase, and a moderate electrical field helps them to overcome the potential barrier. One has to note that interpolaron distances are quite comparable with an electron delocalization length in conjugated polymers. The length was estimated in Ref. 20 for poly(phenylenevinylene)s by using temperature dependence of the magnetic field effect on photoconductivity, and values from 3 to $7 \mathrm{~nm}$ were obtained. It means that an electron escaping from an excited site gets over one or two intermolecular (or interconjugation) distances before reaching a final site in the electron transfer process. One can speculate about the reason of existence of two distinct sizes of polaron pairs. Possibly that fact reflects two possibilities of a charge-transfer process initiated by the light absorption: along and across the conjugated segments in the polymer chain. Though polaron pairs with longer intercharge distance are formed in a much smaller amount, their role in photoconductivity in moderate electrical field can be more noticeable.

\section{CONCLUSION}

We performed ps time-resolved experiments by a twocorrelated-pulse technique on alkyl substituted polythiophene samples. The technique permits studying selectively second order processes between intermediate shortliving species generated by the laser light absorption, which are related to free charge carrier formation. An effect of electrical field applied to a sample have permitted to get evidence on involvment of polaron pairs into the recombination process. It is shown that at high enough excitation power $\left(>25 \mu \mathrm{J} / \mathrm{cm}^{2}\right.$ per a pulse) of the $150 \mathrm{fs}, 400 \mathrm{~nm}$ laser pulses free charge carriers appear as a result of recombination of primarily formed polaron pairs. That process competes with the process of electrical field induced dissociation of polaron pairs. Secondary polaron pairs are shown to be produced in the recombination process, and these pairs, having higher intercharge distances, dissociate into free charge carriers with a high probability. It was revealed that primary polaron pairs are formed with two distinct intercharge distances, of about 4 and $10 \mathrm{~nm}$, and relative amounts of the pairs were determined (about 0.9 and 0.1, respectively). 


\section{ACKNOWLEDGMENTS}

E.L.F. is indebted to Osaka University, Department of Electronic Engineering, for hospitality and acknowledges the support by Grants No. INTAS 97-0992 and RFFI
00-03-32253. This work was partly supported by a Grant-in-Aid for Scientific Research from the Ministry of Culture, Sports, Science and Technology and from the Japan Society for the Promotion of Science (Grant No. 11694156).
${ }^{1}$ Primary Photoexcitations in Conjugated Polymers: Molecular Excitons vs. Semiconductor Band Model, edited by N.S. Sariciftci (World Scientific, Singapore, 1997).

${ }^{2}$ G. Cerullo, S. Stagirs, M. Nisoli, S. De Silvestri, G. Lanzani, G. Kranzelbinder, W. Graupner, and G. Leising, Phys. Rev. B 57, 12806 (1998).

${ }^{3}$ W. Graupner, G. Cerullo, G. Lanzani, M. Nisoli, E.J.W. List, G. Leising, and S. De Silvestri, Phys. Rev. Lett. 81, 3259 (1998).

${ }^{4}$ E.L. Frankevich, A.A. Lymarev, I. Sokolik, F.E. Karasz, S. Blumstengel, R.H. Baughman, and H.H. Horhold, Phys. Rev. B 46, 9320 (1992).

${ }^{5}$ E.M. Conwell and H.A. Mizes, Phys. Rev. B 51, 6953 (1995).

${ }^{6}$ S. Barth and H. Baessler, Phys. Rev. Lett. 79, 4445 (1997).

${ }^{7}$ M. Yan, L.J. Rothberg, F. Papadimitrakopoulos, M.E. Galvin, and T.M. Miller, Phys. Rev. Lett. 72, 1104 (1994).

${ }^{8}$ E. Frankevich, Chem. Phys. Rep. 17, 2069 (1999).

${ }^{9}$ E. Frankevich, H. Ishii, Y. Hamanaka, T. Yokoyama, A. Fujii, S. Li, K. Yoshino, A. Nakamura, and K. Seki, Phys. Rev. B 62, 2505 (2000).

${ }^{10}$ E. Frankevich, R. Hidayat, A. Fujii, M. Ozaki, and K. Yoshino, J.
Soc. Electrical Mat. Eng. 9, 109 (2000).

${ }^{11}$ E.L. Frankevich, A.A. Lymarev, and I.A. Sokolik, Chem. Phys. Lett. 159, 113 (1989).

${ }^{12}$ R. Hidayat, Y. Nishihara, A. Fujii, M. Ozaki, K. Yoshino, and E. Frankevich, Phys. Rev. B 66, 075214 (2002).

${ }^{13}$ R. Sugimoto, S. Takeda, H.B. Gu, and K. Yoshino, Chem. Express 1, 635 (1986).

${ }^{14}$ R. Kersting, U. Lemmer, M. Deussen, H.J. Bakker, R.F. Mahrt, H. Kurz, V.I. Arkhipov, H. Bässler, and E.O. Göbel, Phys. Rev. Lett. 73, 1440 (1994).

${ }^{15}$ L. Onsager, Phys. Rev. 54, 554 (1938).

${ }^{16}$ R.H. Batt, C.L. Braun, and J.F. Hornig, J. Chem. Phys. 49, 667 (1968).

${ }^{17}$ R.R. Chance and C.L. Braun, J. Chem. Phys. 64, 3573 (1976).

${ }^{18}$ P.M. Borsenberger and A.I. Ateya, J. Appl. Phys. 49, 4035 (1978).

${ }^{19}$ T. Kawai, M. Nakazono, R. Sugimoto, and K. Yoshino, J. Phys. Soc. Jpn. 61, 3400 (1992).

${ }^{20}$ E.L. Frankevich and A.A. Lymarev, Mol. Cryst. Liq. Cryst. 218, 103 (1992). 